\title{
STABILITY ANALYSIS OF A DELAY DIFFERENTIAL KALDOR'S MODEL WITH GOVERNMENT POLICIES
}

\author{
CARABALLO, T. and SILVA, A.P.
}

\begin{abstract}
This paper is devoted to analyze the stability of the economy according to an extended version of Kaldor's economic growth model. We consider the role of the government and its simultaneous monetary and fiscal policies and we study whether or not a time delay between the recognition and the implementation of its fiscal policy can affect the economic stability. Numerical simulations provide further conclusions about the long-term behavior of the four variables modeled - namely, national income, capacity of production, bonds value and money supply.
\end{abstract}

Keywords: Economic growth model $\cdot$ Kaldor's model $\cdot$ delay differential equations

AMS Subject Classification: 91B55 $\cdot 91 \mathrm{~B} 62 \cdot 34 \mathrm{D} 20$

\section{Contents}

1. Introduction 1

2. An extended Kaldor's business cycle model with government policies 3

$\begin{array}{lll}\text { 3. Local stability of Kaldor's model } & 8\end{array}$

3.1. Model without delay time 8

3.2. Model with delay time in fiscal policy 12

$\begin{array}{ll}4 . \quad \text { Numerical simulations } & 15\end{array}$

4.1. Investment function given by (11) 16

$\begin{array}{|ll|}4.2 . & \text { Investment function given by } 122\end{array} 20$

5. Conclusions 22

Acknowledgements 24

References 24

\section{Introduction}

In James Tobin's words [15, a contemporaneous economist who defended government intervention to stabilize output and avoid recessions, "the question of 
growth is nothing new but a new disguise for an age-old issue, one which has always intrigued and preoccupied economics: the present versus the future."

Academics 1, 15, 22] and organizations such as World Bank agree that the economic growth and poverty reduction have a positive correlation and hence predicting the former is an important goal to be pursued. As stated in [15], thanks to rising incomes, material standards of living have improved substantially over time for most families in most countries.

Economists have realized throughout the last century how imperative mathematics is for such a goal and often have faced two basic differential equations. We shall denote the national income by $Y$ (sometimes called gross domestic production, GDP), which evolves in time as do the others economic variables, without any further mention. Such a quantity dictates how rich a country is and it grows as long as the nation is capable of accumulating richness, which is possible basically by either increasing the capacity of production or decreasing the amount of money kept under the mattress, and it can be expressed by $Y^{\prime}(t)=\alpha(I(t)-S(t))$, where $I(t)$ and $S(t)$ denote investment and saving, respectively. And implicitly, it has been set that $K^{\prime}(t)=I(t)$, where $K(t)$ is the capacity of production which is called capital stock and which refers to factories, machines, warehouses...

For instance, the IS-LM 15] model is a classical Keynesian one that considers both $I$ and $S$ as linear functions of $Y$ and $K$. It is very handy for didactic purposes but its linear formulation inevitably conducts to only two extreme unrealistic situations: complete economic stability or complete economic instability. As in Kaldor's paper [11, the economy is usually assumed to be closed, that is, there is no trade with other nations. In the early forties, Kaldor was one of the first economists to propose a nonlinear formulation for investment $I$ and saving $S$ as functions of $Y$ and $K$ in order to explain the natural fluctuations of the economy. Kaldor's idea is a tremendous improvement over the IS-LM model and it is invariably present over the last decades: $2,4,6,9,13,19,21,23$. In the sixties, Goodwin [7] proposed a model inspired on Lotka-Volterra equations with the same aim, which also has been studied over the years, as in 5, 17, 18. As pointed out by Matsumoto and Szidarovszky, "nonlinearities and the presence of delay time are the main ingredients for endogenous cycles". This means that these two ingredients should be added in order to obtain a more realistic model.

We propose to continue the analysis started out by Takeuchi and Yamamura 23 who consider extreme cases of an extended version of Kaldor's model that aggregates the government role and its fiscal and monetary policies. To do so, we improve the treatment given by these authors by considering the full version of the model, that is, with four variables instead of three. In the Section 2, after a brief discussion about this extended version, its improvements, its 
restrictions and some assumptions, we prove the existence and uniqueness of a positive equilibrium point. We then establish sufficient conditions so that such a point is asymptotically stable, which can be done with or without delay time on the fiscal policy implementation, as we shall see in Section 3 . For further conclusions, we run simulations and analyze the effect of the fiscal policy strength and the delay time size over the long-term stability in the model. See Section 4. All results, some conclusions and future considerations are included in Section 5.

\section{An extended Kaldor's business cycle model with government policies}

Initially, the basic formulation of Kaldor's model was justified by graph analysis and more than a decade later Ichimura 9] presented a rigorous treatment leading to the following equations

$$
\left\{\begin{array}{l}
Y^{\prime}(t)=\alpha(C(t)+I(t)-Y(t)) \\
K^{\prime}(t)=I(Y(t), K(t))
\end{array},\right.
$$

where $C$ denotes the national consumption. By definition, saving $S$ is the portion of income that it is not spent, $S=Y-C$, so the first equation of (1) can be written as $Y^{\prime}(t)=\alpha(I(t)-S(t))$, that is, the economic growth is proportional to how much investment exceeds saving; and the production capacity growth, $K^{\prime}(t)$, is simply the investment. Thanks to the assumptions (see [11) over the shape of the curves $I$ and $S$, such a model leads to economic oscillations either using the Poincaré-Bendixson theorem as in [4] or using the Hopf bifurcation theorem as in 6]. Ichimura 9] came to the same conclusions thanks to Liénard equation techniques.

Several different delay formulations of (1) have been considered in the last years. For instance, substituting its second equation by

$$
K^{\prime}(t)=I\left(Y\left(t-\tau_{0}\right), K\left(t-\tau_{1}\right)\right)-\delta K(t),
$$

one obtains a formulation that considers the gestation lag of investment and the depreciation effect thanks to the positive parameter $\delta$. The case $\tau_{0}>0=\tau_{1}$ was firstly studied in [13], - where the model is thereafter called the Kaldor-Kalecki model, referring to [11,12 - and more recently in [19], where the authors proved that the dynamic behavior is affected quantitatively by the investment delay but not qualitatively; the case $\tau_{0}=\tau_{1}>0$ was considered by [10], and also by [21], adding a noise perturbation. In 2009, Zhou and Li 25] analyzed a combination of IS-LM and Kaldor's model with two time delays in the capital accumulation processes.

Following [24], Takeuchi and Yamamura [23] added the government and a delay time on its fiscal policy to the model, which were important elements missing, as pointed out in 2, 16. Such a formulation in $\mathbb{R}^{4}$ consists on an adaptation on 
the equations in [11], a government budget constraint and a monetary market equation. To make this precise, we introduce some economic quantities:

(i) the aggregate value of bonds varies with time, so we write $t \mapsto B(t)$. Every bond is assumed to be a consol, that is, a bond with a fixed income security and no maturity date;

(ii) money supply $M(t)$ together with money demand $t \mapsto L(Y(t), M(t))$, which is entirely controlled by the government, are the forces of the money market, in the sense that

$$
M^{\prime}(t)=L(Y(t), M(t))-M(t) ;
$$

(iii) the price level $t \mapsto p(Y(t))$ is an index that corrects the real value of bonds and the money power throughout the time;

(iv) the tax revenue is

$$
t \mapsto T(t)=T(Y(t), B(t))=\theta\left(Y(t)+\frac{B(t)}{p(Y(t))}\right)-T_{0},
$$

where $0<\theta<1$ is the tax rate over the income and the profits on the bonds, and $T_{0}>0$;

(v) government expenditure is

$$
t \mapsto G(t)=G_{0}+\beta\left(Y^{*}-Y(t-\tau)\right),
$$

where $G_{0}$ is the fixed spending and $\beta>0$ measures how the expenditure responds to the excess (or lack) of national income, assuming that the government always know the equilibrium national income $Y^{*}$. The constant delay $\tau \geqslant 0$ represents the policy lag, since it naturally takes time to recognize opportunities to implement a stabilization policy and to actually put it in practice;

(vi) the interest rate of the bonds is $t \mapsto r(Y(t), M(t))$ and it is basically the money price;

Hence the government budget constraint reads as follows:

$$
\frac{M^{\prime}(t)}{p(Y)}+\frac{B^{\prime}(t)}{r(Y, M) p(Y)}=G(t)+\frac{B(t)}{p(Y)}-T(Y, B),
$$

which equates the changes in the stocks of bonds and money to the government deficit, since it is assumed that selling bonds and printing banknotes finance the government deficit. Besides,

(vii) the national consumption is

$$
C(t)=C_{0}+c_{1}\left(Y(t)+\frac{B(t)}{p(Y)}-T(t)\right)+c_{2}\left(\frac{B(t)}{r(Y, M) p(Y)}+\frac{M(t)}{p(Y)}\right),
$$


where $0<c_{1}, c_{2}<1$ are the marginal propensity to consume the available income and the available wealth respectively and $C_{0}>0$ is the minimal (basically vital) consumption; and

(viii) the (nonlinear) investment function $t \mapsto I(Y(t), K(t), M(t))$ represents the amount of money spent on buying goods for future use, which should provide more money.

By considering the money depreciation over time, the variables $B$ and $M$ have to be corrected by the price level, whence the values $Y, C, I, T, G, K, B / p$ and $M / p$ are measured in real terms (let us say, euro or dollar).

An extended version of Kaldor's model in $\mathbb{R}^{4}$ arises by adding (2) and (3) to the original formulation (1) together with the adapted consumption and investment functions and the government expenditure; it reads as follows:

$$
\left\{\begin{array}{l}
Y^{\prime}(t)=\alpha(C(t)+I(t)+G(t)-Y(t)) \\
K^{\prime}(t)=I(Y(t), K(t), M(t)) \\
\frac{M^{\prime}(t)}{p(Y)}+\frac{B^{\prime}(t)}{r(Y, M) p(Y)}=G(t)+\frac{B(t)}{p(Y)}-T(Y, B) \\
M^{\prime}(t)=L(Y, M)-M(t)
\end{array}\right.
$$

On the one hand, fiscal policy refers to the mechanism of increasing or decreasing the expenditure $G$, which directly affects the economic activity, stimulating it or discouraging it, respectively. The government pursues such a policy by adjusting the parameter $\beta$, which is assumed to be positive, since the Kaldor model is essentially a Keynesian one. One could consider that fiscal policy includes the alteration of taxation levels as well, which is achieved by adjusting the parameter $0<\theta<1$. But we do not consider this way because the tax rate $\theta$ is predetermined and nearly unchangeable by political reasons. The immediate consequence of such an assumption is that we do not analyze the stability of the equilibrium point with respect to this parameter.

On the other hand, monetary policy refers to the fact that is the government who effectively prints every banknote in circulation and consequently determines the available money quantity, which affects the price level and the interest rate and consequently investment and national production. These two policies together allow the government to promote economic stability or, unfortunately, instability.

As in 2], Takeuchi and Yamamura considered two extreme scenarios (both lead to an $\mathbb{R}^{3}$ formulation): money finance case by setting $B^{\prime} \equiv 0$ in (4); and bond finance case by setting $M^{\prime} \equiv 0$. In the former, the government controls the money supply but bonds offer keeps constant $B=\bar{B}$; and in the latter, the government controls the bonds supply in order to finance its deficit but it cannot adjust its money supply $(M=\bar{M})$. And then the model stability is analyzed 
under these two settings with or without delay time $\tau$. However such scenarios separately do not fit the practical government activity, therefore we take a step forward by analyzing the model $(4)$ in $\mathbb{R}^{4}$ with its full budget constraint and with or without delay time.

By setting $u=\left(u_{1}, u_{2}, u_{3}, u_{4}\right) \equiv(Y, K, B, M)$ in (4), we obtain

$$
\left\{\begin{aligned}
u_{1}^{\prime}(t)= & \alpha\left(-\left(1-(1-\theta) c_{1}\right) u_{1}(t)-\beta u_{1}(t-\tau)+I(u)\right. \\
& +\frac{\left((1-\theta) c_{1}+c_{2} / r(u)\right) u_{3}(t)+c_{2} u_{4}(t)}{p(u)} \\
& \left.+C_{0}+c_{1} T_{0}+G_{0}+\beta Y^{*}\right) \\
u_{2}^{\prime}(t)= & I(u) \\
u_{3}^{\prime}(t)= & r(u) p(u)\left(-\theta u_{1}(t)-\beta u_{1}(t-\tau)\right. \\
& +\frac{(1-\theta) u_{3}(t)+u_{4}(t)-L(u)}{p(u)} \\
& \left.+G_{0}+\beta Y^{*}+T_{0}\right) \\
u_{4}^{\prime}(t)= & L(u)-u_{4}(t)
\end{aligned}\right.
$$

All the functions are assumed to be as smooth as necessary. Additionally, consider the following assumptions for every $u \in \mathbb{R}_{+}^{4}$ :

(A1) $\left.L(u)\right|_{u_{4}=0}>0, \lim _{u_{4} \rightarrow \infty} L(u)<0$ and $\frac{\partial L}{\partial u_{4}}(u) \leqslant 0<\frac{\partial L}{\partial u_{1}}(u)$;

(A2) $\left.I(u)\right|_{u_{2}=0}>0, \lim _{u_{2} \rightarrow \infty} I(u)<0$ and $\frac{\partial I}{\partial u_{2}}(u)<0<\frac{\partial I}{\partial u_{1}}(u), \frac{\partial I}{\partial u_{4}}(u)$;

(A3) $p(u)>0$ and $\frac{d p}{d u_{1}}(u)>0$; and

(A4) $0<r(u)<1$ and $\frac{\partial r}{\partial u_{4}}(u)<0<\frac{\partial r}{\partial u_{1}}(u)$.

Under these assumptions, we can prove the existence and the uniqueness of a positive equilibrium point.

Assume the government establishes some equilibrium income $Y^{*}>0$ as target and pursues it. By (A1), the right-hand side of the last equation in (5) applied for $u_{1}=Y^{*}$ is a function of $u_{4}$, namely $u_{4} \mapsto L\left(Y^{*}, u_{4}\right)-u_{4}$, such that it is positive for $u_{4}=0$ and it becomes negative as $u_{4}$ increases, since $\frac{\partial L}{\partial u_{4}}(u) \leqslant 0$ 
and $\lim _{u_{4} \rightarrow \infty} L(u)<0$. Thus we obtain a unique value $u_{4}=M^{*}>0$ for which that expression is null.

Setting $u_{1}=Y^{*}$ and $u_{4}=M^{*}$ in the second equation of (4), thanks to (A2), we may argue as before to obtain a unique value $u_{2}=K^{*}>0$ such that $I\left(Y^{*}, K^{*}, M^{*}\right)=0$. Now we set $u_{1}=Y^{*}, u_{2}=K^{*}$ and $u_{4}=M^{*}$ in the first and third equations of (4). Their right-hand sides vanish if and only if

$$
\left\{\begin{array}{l}
0=C\left(Y^{*}, B, M^{*}\right)+G_{0}-Y^{*} \\
0=G_{0}+\frac{B}{p\left(Y^{*}, M^{*}\right)}-T\left(Y^{*}, B\right),
\end{array}\right.
$$

which is a linear system on the variables $B$ and $G_{0}$. So there exists a unique positive equilibrium point $u^{*}=\left(Y^{*}, K^{*}, B^{*}, M^{*}\right)$ if and only if the government can fix a compatible value $G_{0}>0$ so that the system above admits a unique positive solution $B=B^{*}$. It is noteworthy that $u^{*}$ does not depend on $\beta$. Also, about the conditions (A3) and (A4), we just have used the fact that the functions $p$ and $r$ are positive.

Therefore, we have proved the following result.

Lemma 1. Suppose that the conditions $(\boldsymbol{A} 1)-(\boldsymbol{A} 4)$ hold. Given $Y^{*}>0$, if $(6)$ admits a unique positive solution $\left(B^{*}, G_{0}\right)$ then (5) admits a unique positive equilibrium point $u^{*}$ associated to $Y^{*}$, which does not depend on $\beta$.

In 23], under suitable additional technical assumptions one has to deal with expressions where either it is possible to extract a unique positive $Y^{*}$ from one of the equations and then $M^{*}>0$ from other so that $I\left(Y^{*}, K, M^{*}\right)=0$ provides a unique $K^{*}>0$; or by imposing a lower bound to $Y^{*}$ it is possible to obtain $B^{*}>0$ as function of $Y^{*}$ and the remaining argumentation follows analogously.

However we do not have such a scenario, that is, it is not possible to determine a unique $Y^{*}>0$ since every of the four equations of (4) depends nontrivially on at least two variables. Thus we assume that the government is able to establish a national income $Y^{*}>0$ as target and a compatible expenditure value $G_{0}>0$. Doing so, one can obtain a unique associated equilibrium point $u^{*}=\left(Y^{*}, K^{*}, B^{*}, M^{*}\right)$ in $\mathbb{R}_{+}^{4}$ as we did. In our opinion, such a setting is realistic because governments pursue annual growth rates - consequently they reconsider future values of $Y^{*}$ as the economy grows - and they adjust their expenditures and policies accordingly. The reader should recognize now why Lemma 1 requires $\left(B^{*}, G_{0}\right)$ to be positive.

REMARK 2 (About the assumptions). It is natural to expect that the richer a nation the more money it demands; and clearly the money demand $L(u)$ decreases as more money $u_{4}=M$ is provided, whence the derivative assumptions of (A1) are reasonable from the economic point of view. 
As the infrastructure of a nation improves together with its capacity of production - $K_{2}$ increasing - the best opportunities of investment disappear; such a phenomenon is expressed by $\frac{\partial I}{\partial u_{2}}<0$. Besides, investment is stimulated by economic activity and it essentially requires money, whence we unsurprisingly required $\frac{\partial I}{\partial u_{1}}$ and $\frac{\partial I}{\partial u_{4}}$ to be positive.

In capitalist economies, prices rising is an intrinsic reaction to the economic growth, justifying $\frac{d p}{d u_{1}}$ is assumed to be positive. The only point that the government should be concerned about is to keep the associated inflation under control. Clearly $p(u)$ must be positive since it is associated with a weighted mean of all prices practiced in the markets.

The interest rate $r(u)$ is a percentage that defines the remuneration over the money loaned by investors to the government. The reader knows this financial operation by bonds. The more money is available, the smaller is the necessity of the government to be financed by third parties and hence it offers lower remunerations to investors, that is, $\frac{\partial r}{\partial u_{4}}<0$.

Moreover, $\frac{\partial r}{\partial u_{1}}>0$ follows from liquidity preference theory, as in the IS-LM model, which basically states that one dollar today is worth more than one dollar tomorrow. The logic is the following: greater income implies greater money demand which increases the price of money, that is, the interest rate $r$.

The government cannot print banknotes as it pleases it because it would promote a scenario of hyperinflation very hard to handle with and which would immediately cause loss of a prime function of money: store of value. In such an extreme situation, no one wants an additional one dollar bill: money demand is negative! This is expressed by $\lim _{u_{4} \rightarrow \infty} L(u)<0$. Finally, investment refers to the gain of production capacity while depreciation refers to its loss due whether to wear and tear or to obsolete technology. If the production capacity is too high, there is no new investment projects for some time until the point where there is inevitably depreciation; and $\lim _{u_{2} \rightarrow \infty} I(u)<0$ expresses it.

\section{Local stability of Kaldor's model}

Now we analyze the local stability of Kaldor's model (first without delay and later with it) by considering its linearization, as in 8, 14, for the nontrivial equilibrium point $u^{*}$ obtained in Lemma 1 .

3.1. Model without delay time

We shall evaluate the Jacobian matrix for the differential system (5) on $u^{*}$, omitting the argument of functions and its derivatives or even the symbol *. For instance, we simply write $r_{1}$ to denote $\frac{\partial r}{\partial u_{1}}\left(u^{*}\right)$. This minor abuse of notation 
rarely causes problems and it will be very handy for the expressions to come, which will require several renamings.

By (6), if $G^{*}=\left(\theta Y^{*}-G_{0}-T_{0}\right) /(1-\theta)$ then $B^{*}=p^{*} G^{*}$ and the Jacobian matrix evaluated at $u^{*}$ is given by

$$
J=\left(\begin{array}{cccc}
F_{11}(\beta) & \alpha I_{2} & F_{13} & F_{14} \\
I_{1} & I_{2} & 0 & I_{4} \\
F_{31}(\beta) & 0 & F_{33} & r^{*} F_{44} \\
L_{1} & 0 & 0 & -F_{44}
\end{array}\right)
$$

where

$$
\begin{array}{cc}
k_{1}=\alpha\left(1-(1-\theta) c_{1}\right) & k_{2}=\alpha c_{2} \\
b_{11}=k_{1}+k_{2}\left[\frac{r_{1} G^{*}}{\left(r^{*}\right)^{2}}+\frac{\dot{p}^{*} L}{\left(p^{*}\right)^{2}}\right]+\dot{p}^{*} F_{13} G^{*} & b_{31}=r^{*}\left(L_{1}+\theta p^{*}+(1-\theta) \dot{p}^{*} G^{*}\right) \\
F_{11}(\beta):=-\alpha \beta+\alpha I_{1}-b_{11} & F_{31}(\beta):=-p^{*} r^{*} \beta-b_{31} \\
F_{13}=\frac{k_{2}+\left(\alpha-k_{1}\right) r^{*}}{p^{*} r^{*}} & F_{14}=\alpha I_{4}+k_{2}\left[\frac{1}{p^{*}}-\frac{r_{4} G^{*}}{\left(r^{*}\right)^{2}}\right] \\
0<F_{33}=(1-\theta) r^{*}<1 & F_{44}=1-L_{4} .
\end{array}
$$

Furthermore, we write

$$
\begin{array}{cc}
\mu=p^{*} r^{*} F_{13}-\alpha F_{33} & \nu=F_{44}-I_{2} \\
\sigma=\nu-F_{33} & \Gamma=k_{2}\left[\frac{1}{p^{*}}-\frac{r_{4} G^{*}}{\left(r^{*}\right)^{2}}\right]
\end{array}
$$

so that $k_{1}, k_{2}, b_{11}, F_{13}, b_{31}, F_{14}, \Gamma, F_{44}, \nu>0$. The characteristic equation is

$$
\lambda^{4}+a_{1}(\beta) \lambda^{3}+a_{2}(\beta) \lambda^{2}+a_{3}(\beta) \lambda+a_{4}(\beta)=0,
$$

where $a_{j} \equiv a_{j}(\beta):=a_{j 0}+a_{j 1} \beta$, for $j=1,2,3,4$, are given by

- $a_{10}=b_{11}-\alpha I_{1}+\sigma$,

- $a_{11}=\alpha$,

- $a_{20}=-I_{2}\left(\alpha I_{1}+I_{2}\right)+\nu\left(-I_{2}-F_{33}-\frac{F_{14} L_{1}}{\nu}\right)+\sigma\left(b_{11}-\alpha I_{1}\right)+F_{13} b_{31}$,

- $a_{21}=\alpha \nu+\mu$,

- $a_{30}=F_{13} F_{44}\left(b_{31}-r^{*} L_{1}\right)-b_{11} F_{33} I_{2} F_{44}\left[\frac{1}{F_{33}}\left(1-\frac{\Gamma L_{1}}{b_{11} F_{44}}\right)+\frac{1}{I_{2}}-\frac{1}{F_{44}}\right]+$ $F_{33}\left(F_{14} L_{1}+F_{44}\left(\alpha I_{1}+I_{2}\right)\right)-b_{31} I_{2} F_{13}$,

- $a_{31}=-\alpha I_{2} F_{44}+\mu \nu$,

- $a_{40}=-I_{2} F_{13} F_{44}\left(b_{31}-r^{*} L_{1}\right)+b_{11} F_{33} I_{2} F_{44}\left[1-\frac{\Gamma L_{1}}{b_{11} F_{44}}\right]$ and

- $a_{41}=-\mu I_{2} F_{44}$.

We shall analyze whether or not the stability of $u^{*}$ is sensitive with respect to how strong the fiscal policy is, that is, with respect to the parameter $\beta>0$. By Routh-Hurwitz criteria, $u^{*}$ is asymptotically stable if and only if $a_{1}, a_{3}, a_{4}>0$ and $a_{1} a_{2} a_{3}-a_{3}^{2}-a_{1}^{2} a_{4}>0$. 
Lemma 3. Suppose that

(H1) $c_{2}>(1-\theta)\left(1-c_{1}\right) r^{*}$ and $\sigma>0$;

(H2) $F_{33}+F_{14} L_{1} / \nu<-I_{2}<\alpha I_{1}<b_{11}$; and

(H3) $\frac{1}{F_{33}}\left(1-\frac{\Gamma L_{1}}{b_{11} F_{44}}\right)+\frac{1}{I_{2}}-\frac{1}{F_{44}}>0$.

Then

a. if $a_{40} \geqslant 0$ then $a_{j}>0, j=1,2,3,4$ for every $\beta>0$;

b. if $a_{40}<0$ then $a_{j}>0, j=1,2,3,4$ for every $\beta>-a_{40} / a_{41}$.

Moreover, $a_{41}>0$ if and only if $c_{2}>(1-\theta)\left(1-c_{1}\right) r^{*}$.

Proof. It is not hard to see that $\mu=\alpha\left(c_{2}-(1-\theta)\left(1-c_{1}\right) r^{*}\right)$, which is positive by (H1) and then $a_{21}>0$. Also, by (H2)

$$
a_{10}=\underbrace{b_{11}-\alpha I_{1}}_{>0}-I_{>0}-F_{33}+\underbrace{F_{44}}_{>0}>0
$$

and

$$
a_{20}=-I_{2} \underbrace{\left(\alpha I_{1}+I_{2}\right)}_{>0}+\nu \underbrace{\left(-I_{2}-F_{33}-\frac{F_{14} L_{1}}{\nu}\right)}_{>0}+\sigma \underbrace{\left(b_{11}-\alpha I_{1}\right)}_{>0}+F_{13} b_{31}>0,
$$

whence $a_{1}(\beta), a_{2}(\beta)>0$ for every $\beta>0$. Clearly $a_{30}$ and $a_{31}$ are sums of positive terms since $I_{2}<0$; whence $a_{3}(\beta)>0$ for every $\beta>0$. Although $a_{41}>0$ by (H1), $a_{40}$ is a sum of a positive term and a negative one, whence instead of controlling its sign we consider the sign of $a_{4}(\beta)$ for both cases as stated.

REMARK 4. Since $\nu$ is a sum of two (possibly large) positive numbers and $F_{33}$ is a product of two numbers which lie in $(0,1)$, it is not restrictive to assume that $\sigma=\nu-F_{33}>0$ in $(\boldsymbol{H} \mathbf{1})$.

As the reader may promptly realize, even for $a_{j}(\beta)$, which depends linearly on $\beta$, the main challenge is renaming, rearranging and noticing conveniently expressions and hypotheses in order to guarantee the positive sign of large sums and then to fulfill the Routh-Hurwitz conditions. The main theorem below deals with the sign of $p_{R H}(\beta)=a_{1} a_{2} a_{3}-a_{3}^{2}-a_{1}^{2} a_{4}$, which is a cubic function of $\beta$ and which has over 500 terms if it is fully expanded. Although computing systems, such as Wolfram Mathematica, are very handy for symbolic expressions, they are not able to assimilate the concept of 'convenient rearrangements' and hence we must deal with some hard parts by ourselves. 
We may write $p_{R H}(\beta)=Q_{0}+Q_{1} \beta+Q_{2} \beta^{2}+Q_{3} \beta^{3}$, where

$$
\begin{aligned}
& Q_{0}=\frac{a_{30}}{2}\left(a_{10} a_{20}-2 a_{30}\right)+\frac{a_{10}}{2}\left(a_{20} a_{30}-2 a_{10} a_{40}\right), \\
& Q_{1}=a_{31} \underbrace{\left(a_{10} a_{20}-2 a_{30}\right)}_{(\mathbf{E}-\mathbf{1 . 1})}+\alpha \underbrace{\left(a_{20} a_{30}-2 a_{10} a_{40}\right)}_{(\mathbf{E}-\mathbf{1 . 2})}+a_{10} \underbrace{\left(a_{21} a_{30}-a_{10} a_{41}\right)}_{(\mathbf{E}-\mathbf{1 . 3})}, \\
& Q_{2}=a_{10} \underbrace{\left(a_{21} a_{31}-2 \alpha a_{41}\right)}_{(\mathbf{E}-2.1)}+a_{31} \underbrace{\left(\alpha a_{20}-a_{31}\right)}_{\mathbf{( E - 2 . 2})}+\alpha \underbrace{\left(a_{21} a_{30}-\alpha a_{40}\right)}_{\mathbf{( E - 2 . 3})} \text { and } \\
& Q_{3}=\alpha\left(a_{21} a_{31}-\alpha a_{41}\right) .
\end{aligned}
$$

TheOREM 5. Suppose that (E-1.1), (E-1.2), (E-1.3) and (E-2.2) are positive. Under the assumptions of Lemma 3, we have

a. if $a_{40} \geqslant 0$ then $u^{*}$ is asymptotically stable for every $\beta>0$.

b. if $a_{40}<0$ then $u^{*}$ is asymptotically stable for every $\beta>-a_{40} / a_{41}$.

Proof. Note that $Q_{0}$ is a linear combination of (E-1.1) and (E-1.2) with positive weights (under the assumptions of Lemma 3 and that if (E-2.1) is positive then $Q_{3}$ is positive as well. Hence it is sufficient to control the sign of the expressions on $Q_{1}$ and $Q_{2}$ in order to obtain $p_{R H}(\beta)>0$ possibly adding a restriction on $\beta$.

On the one hand, if (H1) holds then

$$
a_{21} a_{31}-2 \alpha a_{41}=\mu^{2} \nu-\alpha^{2} \nu I_{2} F_{44}+\alpha \mu\left(I_{2}^{2}+F_{44}^{2}-I_{2} F_{44}\right)
$$

is a sum of positive terms and (E-2.1) is positive, which immediately implies that $p_{R H}$ is positive for $\beta>0$ large enough. Also, if (H1) holds then (E-2.3) is positive independently of the sign of $a_{40}$ :

$$
\begin{aligned}
a_{21} a_{30}-\alpha a_{40} & >(\mu+\alpha \nu) F_{13} F_{44}\left(b_{31}-r^{*} L_{1}\right)+\alpha I_{2} F_{13} F_{44}\left(b_{31}-r^{*} L_{1}\right) \\
& =F_{13} F_{44}\left(b_{31}-r^{*} L_{1}\right)\left(\mu+\alpha F_{44}-\alpha I_{2}+\alpha I_{2}\right)>0 .
\end{aligned}
$$

On the other hand, let us deal with (E-2.2). From all possible assumptions, the cleanest is $\alpha a_{20}-a_{31}>0$, but we could pursue others conditions. For instance, it is easy to see that $\alpha a_{20}-a_{31}$ is greater than

$$
\alpha \sigma\left(b_{11}-\alpha I_{1}-\frac{I_{2}}{\sigma}\left(\alpha I_{1}+I_{2}-F_{44}\right)\right)+\alpha \nu\left(-I_{2}-F_{33}-\frac{F_{14} L_{1}}{\nu}-\frac{\mu}{\alpha}\right),
$$

which is positive if each term is; note that these two conditions are slightly stronger than (H2), since $I_{2} / \sigma, \mu / \alpha \in(0,1)$. Or yet, $\alpha a_{20}-a_{31}$ is greater than

$$
\alpha \nu\left(-I_{2}-F_{33}-\frac{F_{14} L_{1}}{\nu}+I_{2} \frac{F_{44}}{\nu}-\frac{\mu}{\alpha}\right)
$$

and asking this expression to be positive is again a slightly stronger condition than (H2), since $F_{44} / \nu, \mu / \alpha \in(0,1)$. In both cases, the new conditions are 
considerably larger though. Similarly one can obtain conditions for (E-1.1), (E-1.2), (E-1.3) and (E-2.2) to be positive, where (E-1.1) is the one which demands more effort since it has a longer expression to be dealt with. However we abide by the cleanest assumptions sparing the reader the gruesome estimates and their details; and the proof is complete.

REMARK 6. Actually we proved that if (H1) holds then $Q_{3}>0$ which implies that a strong fiscal policy (that is, a scenario where $\beta>0$ is large enough) always promotes a long-term stable economy, as long as the government does not delay its implementation (since we are dealing with the model without delay so far).

3.2. Model with delay time in fiscal policy

Invariably economic dynamics involves human behavior, which is a decisive factor to be taken into account. It basically refers to the capacity of making decisions after recognizing opportunities and evaluating available resources. Such an aspect can be added appropriately to an economic model by formulating it with delay; that is, instead of considering differential equations where the variables react instantly to external forces independently of the past, a delay formulation does take into account the fact that the past is important when comes to making decisions. A formulation with a nonconstant delay function $t \mapsto \tau(t)$ or considering the government expenditure as function of a weighted average of the national income, let us say $\beta \int_{-\tau(t)}^{0}\left(Y^{*}-Y(s)\right) f(s) d s$, provides a more realistic modeling. We shall analyze the constant delay case.

In (5), delay time $\tau$ models the government capacity of recognizing, formulating and implementing fiscal policies. To obtain such a fixed value $\tau$, one may evaluate the mean policy lag of a nation considering a given period of time. The associated linearized model evaluated at $u^{*}$ is given by $u^{\prime}(t)=J_{0} u(t)+J_{\tau} u(t-\tau)$, where

$$
J_{0}=\left(\begin{array}{cccc}
\alpha I_{1}-b_{11} & I_{2} & F_{13} & F_{14} \\
I_{1} & I_{2} & 0 & I_{4} \\
-b_{31} & 0 & F_{33} & r^{*} F_{44} \\
L_{1} & 0 & 0 & -F_{44}
\end{array}\right) \text { and } J_{\tau}=\left(\begin{array}{cccc}
-\alpha \beta & 0 & 0 & 0 \\
0 & 0 & 0 & 0 \\
-p^{*} r^{*} \beta & 0 & 0 & 0 \\
0 & 0 & 0 & 0
\end{array}\right)
$$

so that $J=J_{0}+J_{\tau}$ and its characteristic equation can be written as

$$
Q_{0}(\lambda)+e^{-\lambda \tau} Q_{\tau}(\lambda)=0,
$$

where

$$
\begin{gathered}
Q_{0}(\lambda):=\lambda^{4}+a_{10} \lambda^{3}+a_{20} \lambda^{2}+a_{30} \lambda+a_{40} \text { and } \\
Q_{\tau}(\lambda ; \beta) \equiv Q_{\tau}(\lambda):=\left(a_{11} \lambda^{3}+a_{21} \lambda^{2}+a_{31} \lambda+a_{41}\right) \beta .
\end{gathered}
$$


By Theorem 5 , the equilibrium point $u^{*}$ is locally stable for every $\beta>0$, whenever $\tau=0$. For $\tau>0$, we know that $u^{*}$ is locally asymptotically stable if and only if every root of (8) has negative real part, see [8, 14]. Also instability is equivalent to the existence of at least one root with positive real part.

REMARK 7. Under the assumptions of Lemma 3, if the additional assumption

(H4) $a_{40}>0$

holds then the real part of every root of $Q_{0}$ is negative, by Routh-Hurwitz criteria.

From now on, we assume that (H1)-(H4) hold. We shall study how the local stability of $u^{*}$ responds to fiscal policy strength, $\beta>0$, and time lag, $\tau$. First we apply the following stability switch result to the delayed model (4).

Theorem 8 (14], Theorem 3.4.1). Consider the equations (8) on $\lambda$ and

$$
F(y):=\left|Q_{0}(i y)\right|^{2}-\left|Q_{\tau}(i y)\right|^{2}=0, \text { for } y \in \mathbb{R} .
$$

Suppose that $\lambda \mapsto Q_{0}(\lambda), Q_{\tau}(\lambda)$ are analytic functions for $\Re \lambda>0$ and that

(i) there is no common pure imaginary roots of $Q_{0}$ and $Q_{\tau}$;

(ii) $\overline{Q_{0}(-i y)}=Q_{0}(i y)$ and $\overline{Q_{\tau}(-i y)}=Q_{\tau}(i y)$, for every $y \in \mathbb{R}$;

(iii) $\lambda=0$ is not a root for (8);

(iv) $\limsup _{\substack{|\lambda| \rightarrow \infty \\ \Re \lambda \geqslant 0}}\left|\frac{Q_{\tau}(\lambda)}{Q_{0}(\lambda)}\right|<1$; and

(v) the equation (9) admits only finitely many real roots.

Then

a. if $F(y)=0$ has no positive roots then no stability switch occurs.

b. if $F(y)=0$ has at least one positive root and each of them is simple then, as $\tau$ increases, a finite number of stability switches occurs and eventually $u^{*}$ becomes unstable.

The assumption (i) holds by Remark 7 and (iii) holds because $a_{4}(\beta)>0$ for every positive $\beta$. Since $Q_{0}$ and $Q_{\lambda}$ are polynomials with real coefficients and $\operatorname{deg} Q_{0}>\operatorname{deg} Q_{\tau}$, assumptions (ii), (iv) and (v) hold. Although $F$ clearly depends on $\beta$, we shall omit such a dependence from time to time. Thus we shall analyze the stability of $u^{*}$ by studying the positive roots of $F(y)=0$. Setting $z=y^{2}$, the function $F$ can be written as

$$
F(z ; \beta) \equiv F(z)=z^{4}+b_{1} z^{3}+b_{2} z^{2}+b_{3} z+b_{4},
$$


where $b_{j} \equiv b_{j}(\beta), j=1,2,3,4$, are given by

$$
\begin{aligned}
& b_{1}(\beta)=a_{10}^{2}-2 a_{20}-a_{11}^{2} \beta^{2}, \\
& b_{2}(\beta)=a_{20}^{2}-2 a_{10} a_{30}+2 a_{40}+\left(2 a_{11} a_{31}-a_{21}^{2}\right) \beta^{2}, \\
& b_{3}(\beta)=a_{30}^{2}-2 a_{20} a_{40}+\left(2 a_{21} a_{41}-a_{31}^{2}\right) \beta^{2} \text { and } \\
& b_{4}(\beta)=a_{40}^{2}-a_{41}^{2} \beta^{2} .
\end{aligned}
$$

Clearly, $F(y)=0$ has no positive roots whenever $F(z)=0$ has no positive roots. Actually, they have the same number of positive simple roots.

It is noteworthy that $b_{4}(\beta)<0$ if and only if $\beta>a_{40} / a_{41}$, and in this case the number of positive roots of $F$ can be 1,2 or 3 only. As we shall see with simulations in Section 4, under a weak fiscal policy scenario - more precisely, for $0<\beta<a_{40} / a_{41}-$, the government efficiency on implementing it does not harm the economic stability because $z \mapsto F(z)$ has no positive zeros. On the other hand, a more careful analysis is required if $\beta>a_{40} / a_{41}$.

Now we discuss the relationship between the parameters $\beta$ and $\tau$. First note that a pure complex number $\lambda=i y$, where $y>0$, is a root of (8) if and only if $y$ is a positive root of $(9)$.

We shall show only the sufficiency in order to fix some notations. Note that $\left|Q_{0}(i y) / Q_{\tau}(i y)\right|=1$ so that there exists a unique value $\phi(y) \in[0,2 \pi)$ such that $-e^{-i \phi(y)}=Q_{0}(i y) / Q_{\tau}(i y)$ and hence $\lambda=i y$ is a root of (8) whenever $\tau$ is of the form $(\phi(y)+2 n \pi) / y$, with $n \in \mathbb{Z}_{+}$.

The reader should promptly see that, by hypothesis (i) of Theorem $8, Q_{\tau}(i y)$ cannot be zero, whenever $i y$ is a root of (8) or $y$ is a root of $(9)$. If we write $Q_{l}(i y)=Q_{l, \Re}(y)+i Q_{l, \Im}(y), l=0, \tau$, then after some computations we see that $\phi(y) \in[0,2 \pi)$ is the angle that satisfies the equations

$$
\left\{\begin{array}{l}
\cos (\phi(y))=-\frac{Q_{0, \Re}(y) Q_{\tau, \Re}(y)+Q_{0, \Im}(y) Q_{\tau, \Im}(y)}{\left|Q_{\tau}(y)\right|^{2}}=:-\frac{A(y)}{\left|Q_{\tau}(y)\right|^{2}} \\
\sin (\phi(y))=\frac{-Q_{0, \Re}(y) Q_{\tau, \Im}(y)+Q_{0, \Im}(y) Q_{\tau, \Re}(y)}{\left|Q_{\tau}(y)\right|^{2}}=: \frac{B(y)}{\left|Q_{\tau}(y)\right|^{2}}
\end{array},\right.
$$

so that $(0, \infty) \ni y \mapsto \phi(y) \in(0,2 \pi)$ is defined by

$$
\phi(y)=\left\{\begin{array}{cl}
\arctan \left(\frac{-B(y)}{A(y)}\right), & \text { if } \cos (\phi), \sin (\phi)>0 \\
\pi / 2, & \text { if } \cos (\phi)=0 \text { and } \sin (\phi)=1 \\
\pi+\arctan \left(\frac{-B(y)}{A(y)}\right), & \text { if } \cos (\phi)<0 \\
3 \pi / 2, & \text { if } \cos (\phi)=0 \text { and } \sin (\phi)=-1 \\
2 \pi+\arctan \left(\frac{-B(y)}{A(y)}\right), & \text { if } \cos (\phi)>0 \text { and } \sin (\phi)<0
\end{array} .\right.
$$


We regard the root of (8) as a function of $\tau$ by writing $\tau \mapsto \lambda(\tau)=x(\tau)+i y(\tau)$ and then we study the sign of the derivative of $\Re \lambda(\tau)$ at the points where $\lambda(\tau)$ is purely imaginary, which are precisely where a stability switch may occur, since $\lambda=0$ is not a root of (8). Arguing as in the proof of Theorem 8 , we see that $\lambda(\tau)$ is differentiable at $\tau=\tau^{*}$ whenever $\lambda\left(\tau^{*}\right)$ is a simple root. And if, in addition, $\lambda\left(\tau^{*}\right)=i y\left(\tau^{*}\right)$ then we explicitly obtain

$$
\left(\left.\frac{d \lambda(\tau)}{d \tau}\right|_{\tau=\tau^{*}}\right)^{-1}=\left.\left[-\frac{Q_{0}^{\prime}(\lambda)}{\lambda Q_{0}(\lambda)}+\frac{Q_{\tau}^{\prime}(\lambda)}{\lambda Q_{\tau}(\lambda)}-\frac{\tau}{\lambda}\right]\right|_{\tau=\tau^{*}}
$$

so we can determine the direction of motion of $x(\tau)$ as $\tau$ passes through $\tau^{*}$ according to

$$
S:=\left.\operatorname{sign} \frac{d \Re \lambda(\tau)}{d \tau}\right|_{\tau=\tau^{*}}=\left.\operatorname{sign} \frac{d F(y)}{d y}\right|_{y=y\left(\tau^{*}\right)} .
$$

Lemma 9 (14, Theorem 3.4.1). If $y^{*}$ is a simple positive root of 9 then there exists a pair of simple conjugate pure imaginary roots $\lambda\left(\tau^{*}\right)= \pm i y\left(\tau^{*}\right)$ of (8) at $\tau^{*}=\phi\left(y^{*}\right) / y^{*}$ which crosses the imaginary axis according to (10). More precisely,

a. if $S>0$ then $\lambda(\tau)$ crosses the imaginary axis at $\tau=\tau^{*}$ from left to right, that is, $u^{*}$ becomes unstable; and

b. if $S<0$ then $\lambda(\tau)$ crosses the imaginary axis at $\tau=\tau^{*}$ from right to left, that is, $u^{*}$ becomes stable.

If $y_{1}^{*}>\cdots>y_{m}^{*}>0$ are the simple positive roots of (9) then, by making explicit the dependence on $\beta$, we write

$$
S_{i, n}(\tau):=\tau-\frac{\phi\left(y_{i}^{*}(\beta)\right)+2 n \pi}{y_{i}^{*}(\beta)},
$$

for $i=1, \ldots, m$ and $n \in \mathbb{Z}_{+}$. It is an auxiliary function whose zero is the value $\tau$ at which $\lambda(\tau)$ crosses the imaginary axis. These are the tools needed to study numerically the stability switch.

\section{Numerical simulations}

First, we emphasize that $u^{*}$ does not depend on $\beta$ but its stability may. By running simulations in Wolfram Mathematica 11.3, we shall analyze several aspects: how the number of positive simple roots of $F(z ; \beta)=0$ changes as $\beta$ varies; how the convergence of the solution responds to greater values of $\tau$, with eventual instability; the sensitiveness of hypotheses with respect to economic parameters; the stability region in the $\beta \tau$-plane; and so on. For $u=\left(u_{1}, u_{2}, u_{3}, u_{4}\right) \in \mathbb{R}_{+}^{4}$, set 


$$
\begin{array}{lll}
\alpha=0.40 & C_{0}=10 & p(u)=0.4 u_{1}+10 \\
c_{1}=0.40 & T_{0}=20 & r(u)=\frac{1+u_{1}}{1+u_{1}+5 u_{4}} \\
c_{2}=0.15 & \theta=0.35 & L(u)=5 u_{1}-u_{4}+50
\end{array}
$$

As for the investment function, we consider two different formulations, namely,

$$
I(u)=-0.25 u_{2}+5 r(u) u_{4}+100
$$

and

$$
\Im(u)=\eta \tilde{I}(u)+(1-\eta) I(u),
$$

where $\eta \in(0,1)$ is fixed and

$$
\tilde{I}\left(u_{1}, u_{2}\right):=25 \exp \left(\frac{-\log 2}{\left(\frac{15}{1000} u_{1}+10^{-5}\right)^{2}}\right)+\frac{u_{1}}{100}+5 \frac{320^{3}}{\left(u_{2}+1\right)^{3}} .
$$

The first one satisfies the original assumptions of Kaldor's paper [11 concerning the nonlinearity of $I$ with respect to $u_{1}=Y$ but not with respect to $u_{2}=K$. For numerical simulations and to verify the sufficient assumptions, the linear dependence on $u_{2}$ is convenient though. The second one is an adaption of the investment function which appears in 19] and it completely satisfies Kaldor's assumptions over the shape of $I$ curve with respect to $u_{1}=Y$ and $u_{2}=K$.

We shall consider two subsections for the simulations accordingly to the choice of investment function.

REMARK 10. The delay equations demand a function $\psi:[-\tau, 0] \rightarrow \mathbb{R}^{4}$ as initial data. In the simulations, we considered exponential functions with a slow increasing rate, for instance $t \mapsto \exp (0.02 t) 25$ for $u_{1}(t)$. Not that an economy increases indefinitely exponentially on time, but in short-time it is reasonable that a nation has an economic growth of $2 \%$ and that is precisely the point.

4.1. Investment function given by (11)

The economic assumptions (A1)-(A4) and the technical hypotheses (H1)-(H3) are satisfied. If the government pursues the national income $Y^{*}=100$ then it must fix $G_{0}=G\left(u^{*}\right)=0.51$ to obtain $u^{*}=(100.00,776.36,1114.68,275.00)$ as the unique positive equilibrium point of (4), which is asymptotically stable for every $\beta>0$, by Lemma 3 and Theorem 5. In other words, in such a scenario, assuming that the government instantly applies its fiscal policies then no matter how strong they are, the economy is always stable.

First, we set $\beta=0.40$, then the eigenvalues of the associated Jacobian matrix at $u^{*}$ are

$$
-2.2112,-0.0415 \text { and }-0.2097 \pm 0.3409 i
$$


and we obtain the graphs in Fig. 1 comparing the numerical solutions for the model with or without delay of $\tau=10$, represented by the dashed and continuous lines, respectively.

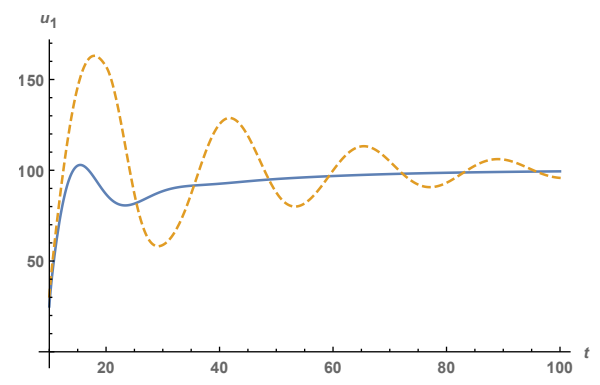

(A) The national income $u_{1}=Y$.

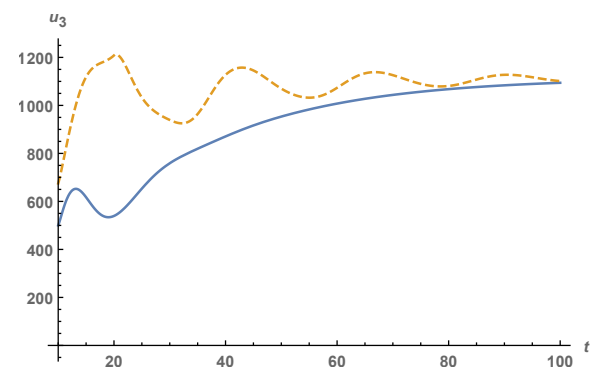

(C) The governments bonds $u_{3}=B$.

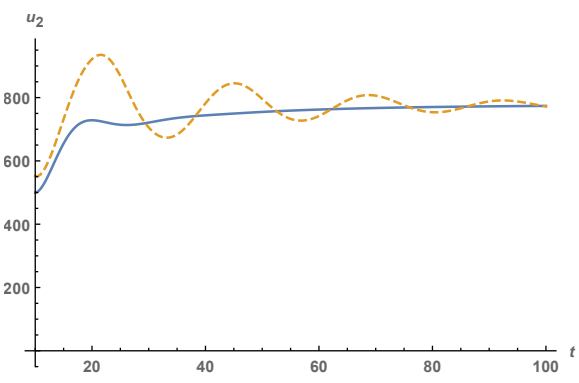

(в) The capital stock $u_{2}=K$.

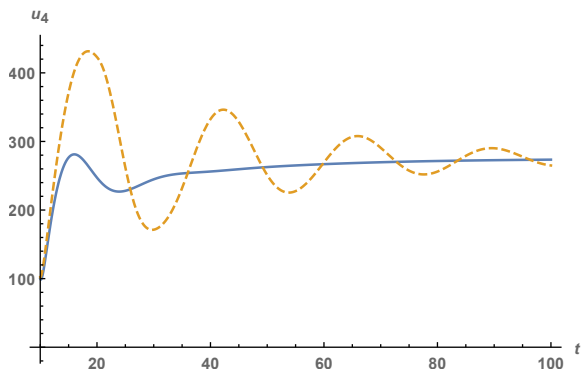

(D) The money supply $u_{4}=M$.

Figure 1. The evolution in time with and without delay.

We have $T\left(u^{*}\right)=22.80$, which says that in equilibrium the government revenue represents about $20 \%$ of the national richness, a compatible idea with the capitalist philosophy about a moderate size for the state accounts. Also, it is reasonable that an interest rate of $r^{*}=6.84 \%$ in equilibrium promotes high values in the bonds market, that is, $B^{*}=1114.68$.

In Fig. 2, we compare the numerical solutions of $u_{1}$ associated to $\tau=0$ (the continuous line), $\tau=10$ (the dotted line) and $\tau=25$ (the dashed line). As $\tau$ increases the solution associated to its delay equation becomes more erratic but $u_{1}^{*}=100$ still is asymptotically stable for $\tau=25$; actually even for $\tau=50$.

However, since $a_{40}=0.0048$, Theorem 8 holds and then we shall study how $\tau$ affects the stability of (4). If $N(\beta)$ denotes the number of positive simple roots 


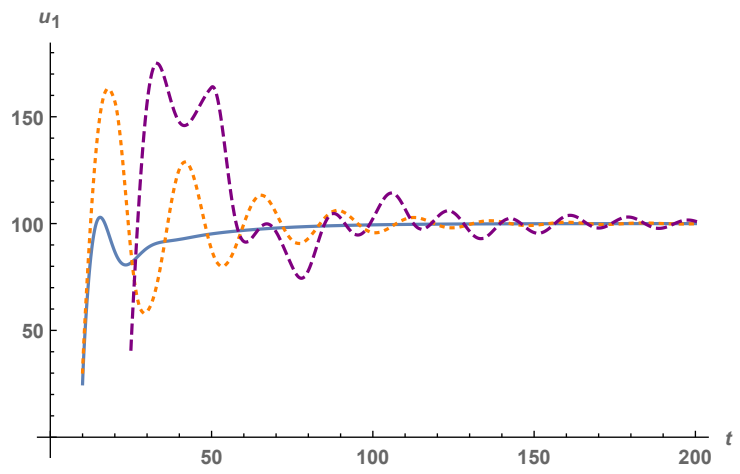

Figure 2. The evolution of $u_{1}(t)$ under different values of $\tau$.

of $F(y ; \beta)=0$ then

$$
N(\beta)=\left\{\begin{array}{ll}
0, & \text { if } 0<\beta<0.1964 \\
1, & \text { if } 0.1964<\beta<0.6071 \text { or } \beta>0.7790 \\
3, & \text { if } 0.6071<\beta<0.7790
\end{array} .\right.
$$

The unique positive simple root of $F(y ; 0.40)=0$ is $y^{*}=0.035472$ and the derivative $\frac{d}{d y} F(y ; 0.40)$ is always positive for $y>0$, whence the crossing the imaginary axis is always to the right half-plane, that is, stability switch occurs only toward instability. The evolution of $u_{1}$ for $\tau=100$ is showed in Fig. 3. Actually, Fig. 4a points out that the stability switch already occurs toward instability at $\tau=67.28$.

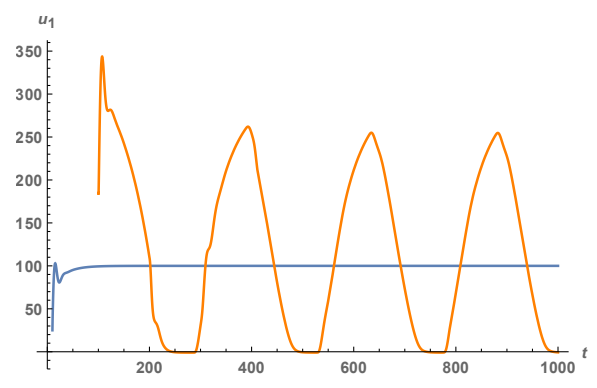

(A) Until $t=1000$.

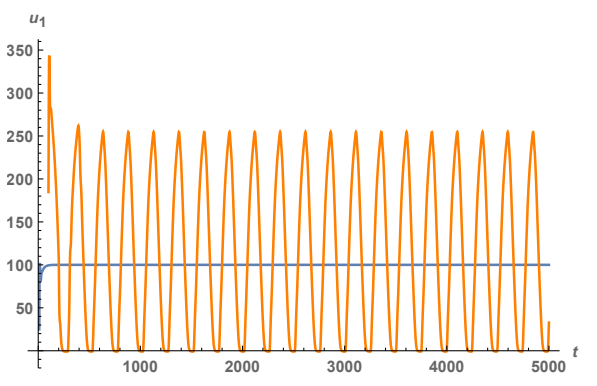

(B) Until $t=5000$.

FIGURE 3. The evolution of $u_{1}$ with $\beta=0.40$ and $\tau=100$. 
On the other hand, the switch stability analysis for $\beta=0.70$ is far more involving since now we have three positive simple roots of $F(y ; 0.70)=0$, namely, $y_{1}^{*}=0.4010, y_{2}^{*}=0.2146$ and $y_{3}^{*}=0.0878$. Besides, $\frac{d}{d y} F(y ; 0.70)$ is negative if $0.1599<y<0.3432$ and it is positive otherwise; whence it follows that $\frac{d}{d y} F\left(y_{1}^{*} ; 0.70\right)$ and $\frac{d}{d y} F\left(y_{3}^{*} ; 0.70\right)$ are positive but $\frac{d}{d y} F\left(y_{2}^{*} ; 0.70\right)<0$. Thus the crossing at $i y_{1}^{*}$ and $i y_{3}^{*}$ must be to the right half-plane; and the crossing at $i y_{2}^{*}$ must be to the left half-plane.

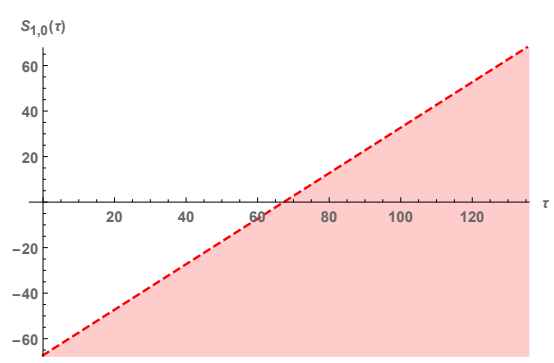

(A) For $\beta=0.40, u^{*}$ becomes unstable at $\tau=67.28$.

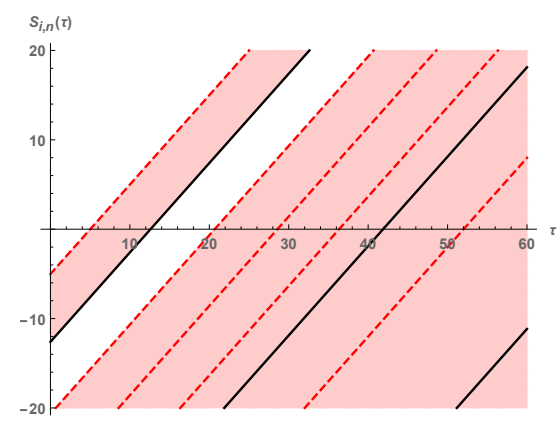

(B) For $\beta=0.70, u^{*}$ becomes unstable as $\tau$ enters in some shaded area and it becomes stable as $\tau$ leaves some shaded area.

Figure 4 . The auxiliary function $S_{i, n}$ and the stability switch of $u^{*}$.

Recall that (8) admits infinitely many complex roots $\lambda(\tau)$. Let $\tau_{i, n}$ be the zero of $S_{i, n}$. In Fig. $4 \mathrm{~b}$ the interceptions of the lines with the $\tau$-axis are at $\tau_{1,0}<\tau_{2,0}<\tau_{1,1}<\tau_{3,0}<\tau_{1,2}<\tau_{2,1}<\tau_{1,3}<\ldots$ and we see how the stability of $u^{*}$ changes as $\tau$ increases. At $\tau=\tau_{1,0}=5.01$, one of the roots of (8) crosses to the right half-plane and then the stability switch occurs toward instability; at the second value $\tau=\tau_{2,0}=12.60$, such a root crosses back to the left half-plane and the switch occurs toward stability. As one may note, as $\tau$ increases, passing by $\tau_{1,1}, \tau_{3,0}$ and $\tau_{1,2}$, three roots of (8) cross to the right half-plane but only one of them crosses back to the left half-plane at $\tau_{2,1}=41.88$ and the instability persists thereafter because the number of roots crossing to the right half-plane exceeds the number of those crossing back.

Finally, by setting $\beta=0.15$, we have that $b_{4}(0.15)>0$ and all roots of $F(y)=0$ are complex, consequently $u^{*}$ is (locally) asymptotically stable for every time delay $\tau>0$. All such conclusions are summarized in Fig. 5 , which 
shows the relationship between the stability of the equilibrium point $u^{*}$ and the parameters $\beta$ and $\tau$.

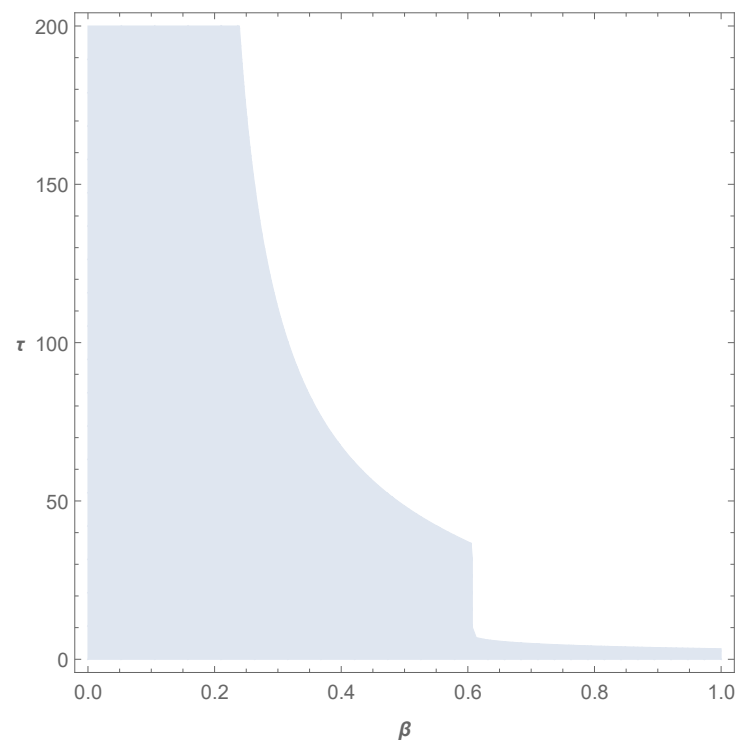

FigURE 5. Stability of $u^{*}$ with respect to $\beta$ and $\tau$.

The boundary of the region consists of the pairs $(\beta, \tau)$ such that $\tau=\tau(\beta)$ is the smallest delay time at which a stability switch occurs toward instability. In the shaded region, $u^{*}$ is asymptotically stable and out of it, there are three possibilities. If $\beta<a_{40} / a_{41}=0.1964$ then $N(\beta)=0$, which means that no stability switch occurs. If $0.6071<\beta<0.7790$ then finitely many stability switches occur as $\tau$ increases, as we discussed above. If $\beta$ is greater than 0.1964 but is not in $(0.6071,0.7790)$, we have instability for every $\tau$ such that $(\beta, \tau)$ lays outside the shaded region.

\subsection{Investment function given by 12}

Let $\eta=0.4, \alpha=0.2$ and consider the investment function in 12 .

In this setting, the economic assumptions (A1)-(A4) and the technical hypotheses (H1)-(H4) are satisfied. Although (E-1.1),(E-1.2) and (E-1.3) are positive, unfortunately (E-2.2) is negative (even after several attempts with different values of parameters) and hence we cannot apply Theorem 5 . However we can verify numerically that the maximum value of the real part of the eigenvalues of the Jacobian matrix at the equilibrium point is negative for every $0<\beta<1$. 
Thus Kaldor's model (4), with $\tau=0$, is locally asymptotically stable even without fulfilling the conditions of our results, making explicit the fact that they are not necessary. Furthermore, we can verify that Theorem 8 holds because, for every $0<\beta<1$, there is no common pure imaginary roots of $Q_{0}$ and $Q_{\tau}$ and $\lambda=0$ is not a root for 8 .

REMARK 11. We have not been able to establish general necessary conditions to existence of a positive equilibrium point. However, we know that $a_{41}$ is positive if and only if $c_{2}>(1-\theta)\left(1-c_{1}\right) r^{*}$, see Lemma 3; whence it is a necessary condition to fulfill Routh-Hurwitz criteria. This very same condition was already required by [23] in Theorem 3.2 to obtain the stability of the equilibrium point.

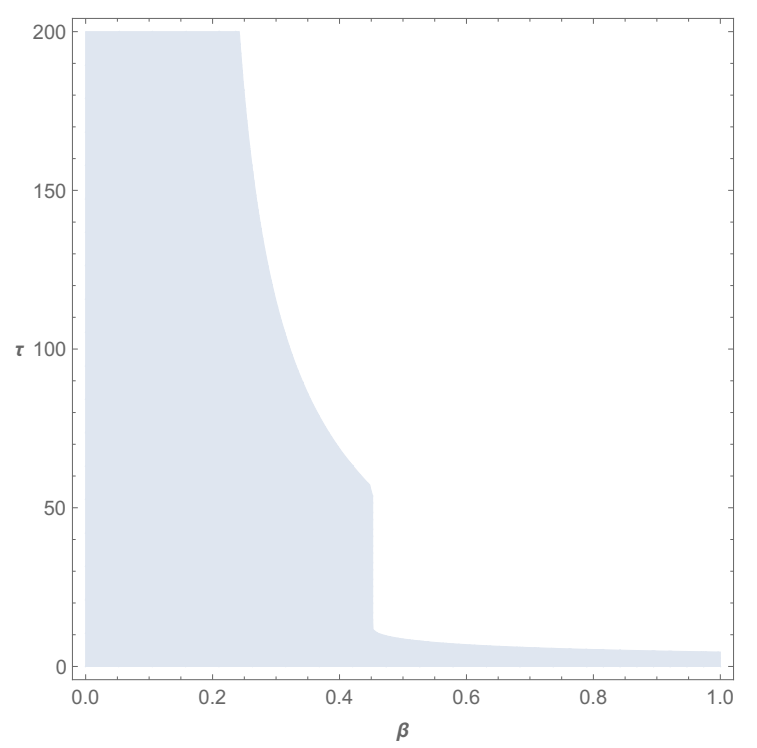

Figure 6. Stability of $u^{*}$ with respect to $\beta$ and $\tau$.

Proceeding as before, we have that

$$
N(\beta)= \begin{cases}0, & \text { if } 0<\beta<0.1964 \\ 1, & \text { if } 0.1964<\beta<0.4523 \text { or } \beta>0.5980 \\ 3, & \text { if } 0.4523<\beta<0.5980\end{cases}
$$

and the region of stability of $u^{*}$ in the $\beta \tau$-plane is given by Fig. 6. Under a moderate fiscal policy, $\beta=0.30$, the government inefficiency does not harm the economic stability until $\tau_{1,0}=115.19$, see Fig.7a. On the other hand, if the fiscal 
policy is slightly stronger, let us say $\beta=0.48$, several switch stabilities occur as $\tau$ increases. More precisely, at $\tau_{1,0}=9.36$, the stability switch occurs toward instability; at the second value $\tau_{2,0}=15.63$, the switch occurs toward stability and so on, depending on whether $\tau=\tau_{2, n}$ or $\tau=\tau_{1, n}, \tau_{3, n}$. See Fig. 7b, where $\tau_{1,0}<\tau_{2,0}<\tau_{1,1}<\tau_{3,0}<\tau_{2,1}<\tau_{1,2}<\tau_{2,2}<\tau_{1,3}<\ldots$

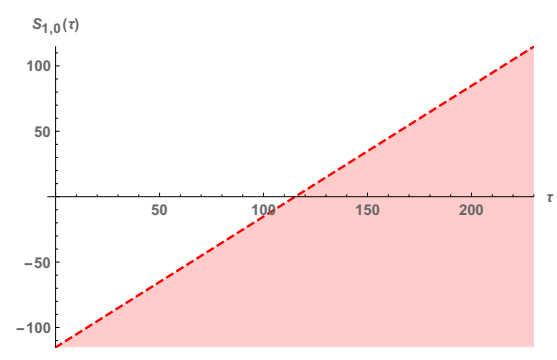

(A) For $\beta=0.30, u^{*}$ becomes unstable at $\tau=115.19$.

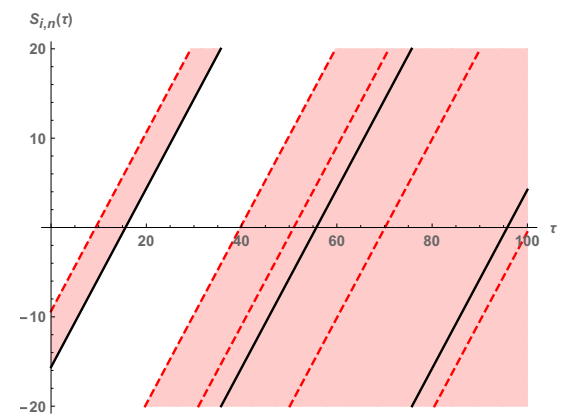

(B) For $\beta=0.48: u^{*}$ becomes unstable as $\tau$ passes by some dashed (red) line and it becomes stable as $\tau$ passes by some continuous (black) line.

Figure 7. The stability switch of $u^{*}$.

\section{Conclusions}

We consider an extended version of the classical Kaldor's economic growth model adding the government role to the economic dynamics: monetary and fiscal policies and the government budget constraint are taken into account, leading to a differential system in $\mathbb{R}^{4}$, with or without a delay time on the fiscal policy. An analysis of the model stated in $(5]$ is itself an improvement over 23 who turned it into two simpler versions in $\mathbb{R}^{3}$ by imposing either $B^{\prime}=0$ or $\bar{M}^{\prime}=0$, that is, extreme scenarios where either the government is incapable to manage its bonds supply or it is incapable to establish its money supply.

Firstly we have proved the existence and uniqueness of a positive equilibrium point under reasonable economic assumptions (which represent an improvement over those technical ones required by 23$]$ ). Secondly we have established sufficient conditions under which (5), with $\tau=0$, is locally asymptotically stable with a possible restriction over the fiscal policy strength. Under a simple additional assumption, namely (H4), we have applied a classical stability switch 
result to study how the fiscal policy delay time may lead to an instable economic scenario.

In Section 4 we have run simulations with two different investment functions, splitting it into two subsections. On the one hand, all assumptions needed for the results we have presented are satisfied by the investment function (11) and by the other functions and parameters. Table 1 summarizes the results of Subsection 4.1

\begin{tabular}{|c|c|c|c|}
\hline$\beta$ & $\begin{array}{c}\text { Strength of the } \\
\text { fiscal policy }\end{array}$ & $\begin{array}{c}\text { First value } \tau \\
\text { under which } u^{*} \\
\text { is unstable }\end{array}$ & Conclusion \\
\hline 0.15 & weak & $\infty$ & $\begin{array}{c}\text { the economy is } \\
\text { always stable }\end{array}$ \\
\hline 0.40 & moderate & 67.28 & $\begin{array}{c}\text { an inefficient government } \\
\text { can lead the economy } \\
\text { to instability }\end{array}$ \\
\hline 0.70 & strong & 5.01 & $\begin{array}{c}\text { the economic stability } \\
\text { is very sensitive to the } \\
\text { government efficiency }\end{array}$ \\
\hline
\end{tabular}

TABLE 1. The effects of the fiscal policy on the economy.

Here government efficiency refers to the time efficiency on recognizing opportunities to implement a fiscal policy, formulating it and then implementing it. Curiously, if the fiscal policy is very strong, let us say $\beta=0.9$, the conclusion are quite the same as those for $\beta=0.4$.

On the other hand, in Subsection 4.2, the investment function is a convex combination of the previous one with the investment function suggested by [19]. Although we cannot apply Theorem 5 for $\alpha=0.2$, we were able to verify numerically that the equilibrium point is always locally asymptotically stable for $0<\beta<1$ and $\tau=0$; and that the switch stability theorem holds as well.

The less simplifications are imposed and the more relevant aspects are considered, the more realistic a model is. For instance, one should expect that the government capacity of recognizing, formulating and implementing fiscal policies varies with time, that is, it is more reasonable to assume a delay function $t \mapsto \tau(t)$ instead of a fixed delay time. Also the economy intrinsically carries a volatility which comes from the human behavior factor and which can be appropriately added to the model by considering certain economic parameters random. For 
instance, $0<c_{1}, c_{2}<1$ dictate how big is the portion of the income that will be spent, which are associated with the (microeconomic) perception whether or not the economy prospers and it will continue to do so. And as we have discussed in Section 2, one could aggregate a delayed investment formulation of KaldorKalecki's model suitably adapted; as in $[19]$. Besides, a question of structural stability arises. Comparing (1) and (4), one may wonder if the limit cycle structure of (1) is present in the extended model. More precisely, is it possible to obtain the original $\mathbb{R}^{2}$ dynamics from (4) by deforming it appropriately?

Our future aims concerns these subjects and other related ones.

\section{Acknowledgements}

The author Tomás Caraballo wishes to thank to Ministerio de Economía y Competitividad (Spain) and to FEDER (European Community), grant MTM201563723-P (MINECO, FEDER, EU) and to Junta de Andalucía, proyecto P12FQM-1492. And the author Alex P. da Silva wishes to thank to Coordenação de Aperfeiçoamento de Pessoal de Nível Superior, grant PROEX-6422328/D and PDSE 132006/2016-01, by financial support.

\section{References}

1] Robert J. Barro and Xavier Sala-i-Martin, Economic growth, McGraw-Hill, 2004

[2] Alan S Blinder, Robert M Solow, et al., Does fiscal policy matter?, Econometric Research Program, Princeton University, 1973.

[3] Luigi De Cesare and Mario Sportelli, A dynamic IS-LM model with delayed taxation revenues, Chaos, Solitons \& Fractals 25 (2005), no. 1, $233-244$.

[4] W.W. Chang and D.J. Smyth, The existence and persistence of cycles in a non-linear model: Kaldor's 1940 model re-examined, The Review of Economic Studies 38 (1971), no. 1, 37-44.

[5] Günter Gabisch and Hans-Walter Lorenz, Business cycle theory. a survey of methods and concepts, 2nd ed., Springer-Verlag Berlin Heidelberg, 1989.

[6] G. Gandolfo, Economic dynamics: Study edition, Economic Dynamics, Springer, 1997.

[7] Richard M. Goodwin, A growth cycle, C.H.Feinstein(Ed.), Socialism, Capitalism and Economics. Cambridge University Press (1967), 54-58.

[8] Jack K. Hale and Sjoerd M. Verduyn Lunel, Introduction to functional-differential equations, Applied Mathematical Sciences, vol. 99, Springer-Verlag, New York, 1993.

[9] S. Ichimura, Toward a general nonlinear macrodynamic theory of economic fluctuations, Post-Keynesian Economics (1955), 192-226.

[10] Abdelilah Kaddar and H Talibi Alaoui, Hopf bifurcation analysis in a delayed KaldorKalecki model of business cycle 13 (2008), 439-449.

[11] Nicholas Kaldor, A model of the trade cycle, The Economic Journal 50 (1940), no. 197, 78-92.

[12] M. Kalecki, A macrodynamic theory of business cycles, Econometrica 3 (1935), no. 3, $327-344$.

[13] A. Krawiec and M. Szydlowski, The Kaldor-Kalecki business cycle model, Annals of Operations Research 89 (1999), no. 0, 89-100. 
[14] Yang Kuang, Delay differential equations: With applications in population dynamics, Mathematics in Science and Engineering, Elsevier Science, 1993.

[15] Nicholas Mankiw, Principles of economics, 5th ed., South-Western Cengage Learning, South-Western Cengage Learning, 2011.

[16] Akio Matsumoto, Destabilizing effects on income adjustment process with fiscal policy lags, Metroeconomica 59, no. 4, 713-735.

[17] Akio Matsumoto, Ugo Merlone, and Ferenc Szidarovszky, Goodwin accelerator model revisited with fixed time delays, Communications in Nonlinear Science and Numerical Simulation 58 (2018), 233 -248. Special Issue on.

[18] Akio Matsumoto, Keiko Nakayama, and Ferenc Szidarovszky, Goodwin accelerator model revisited with piecewise linear delay investment 08 (2018), 178-217.

[19] Akio Matsumoto, F. Szidarovszky, and T. Asada, Essays in economic dynamics: Theory, simulation analysis, and methodological study, Springer Singapore, 2016.

[20] Akio Matsumoto and Ferenc Szidarovszky, Delay dynamics in a classical is-lm model with tax collections., Metroeconomica 67 (2016), no. 4, $667-697$.

[21] Gabriela Mircea, Mihaela Neamtu, and Dumitru Opris, The Kaldor-Kalecki stochastic model of business cycle, Nonlinear Analysis 16 (2011), no. 2, 191-205.

[22] Xavier Sala-i-Martin, The world distribution of income: Falling poverty and ... convergence, period, The Quarterly Journal of Economics 121 (2006), no. 2, 351-397.

[23] Yasuhiro Takeuchi and Tatsuya Yamamura, Stability analysis of the Kaldor model with time delays: monetary policy and government budget constraint, Nonlinear Anal. Real World Appl. 5 (2004), no. 2, 277-308.

[24] Elmar Wolfstetter, Fiscal policy and the classical growth cycle, Zeitschrift für Nationalökonomie 42 (1982), no. 4, 375-393.

[25] Lujun Zhou and Yaqiong Li, A dynamic IS-LM business cycle model with two time delays in capital accumulation equation, Journal of Computational and Applied Mathematics 228 (2009), no. 1, $182-187$.

Departamento de Ecuaciones DiferenCIALES y ANÁLisis NumÉrico FACUlTAD DE MATEMÁticas - UniversiDAD DE SEvilla

Calle Tarfia s/n, 41012 Sevilla, Spain CARABALL@US.ES
Instituto de CiênCias Matemáticas e DE Computação

Universidade de São Paulo, 13566-590

São Carlos, Brazil

ALEXPS@ICMC.USP.BR 